\title{
Author Index of Volume 220 Issue 1
}

Abney, K. D., 12

Adam, A., 67

Aechter, B., 107, 110

Andara, A., 5

Babizhetskyy, V., 1

Bao, R.-L., 85

Beck, W., 107, 110

Ben Nasr, C., 105

Boubekeur, K., 71

Brito, I., 87

Brockner, W., 67

Cai, G.-Q., 69

Cao, S.-J., 119

Cassels, B. K., 79

Chaban, N., 1

Chan, B. C., 7, 9, 11, 12, 13

Chen, B., 81

Chen, Z.-X., 85

Císałová, I., 113

Cordier, S., 116

Dai, J.-C., 93

Ding, J.-C., 23

Ding, M.-W., 119

Dorhout, P. K., 7, 9, 11, 12, 13

Duewel, M., 67

Eger, R., 3

Fan, J., 58, 61

Fan, S.-R., 69

Fan, X., 25

Fang, X.-N., 75

Fässler, T. F., 49, 53

Feng, P. L., 9, 11, 12, 13

Feng, W., 23

Feng, Y.-L., 97, 99

Fernández-González, Á., 5

Fukuda, Y., 31

Galdámez, A., 79

Garbarczyk, J., 103

García-Granda, S., 5

Gholivand, K., 65

Gjikaj, M., 67

Golhen, S., 116

Han, L.-J., 25

Havlí̌cek, V., 113

Hess, R. F., 12

Hoch, C., 3

Hoffmann, H. M. R., 89

Hoffmann, S. D., 49, 53

Horiuchi, A., 27

Horiuchi, C. A., 27

Horn, E., 27, 29, 31

Hosseinian, A., 47

Huang, C.-F., 75

Hulvey, Z., 9, 11, 12

Hušák, M., 113
Ito, K., 27

Iturriaga-Vásquez, P., 79

Jafarpour, F., 15

Jansen, M., 95

Jegorov, A., 113

Jiang, L., 85

Jiang, M.-H., 101

Kim, B. G., 21

Kirakci, K., 116

Knizek, J., 107, 110

Köckerling, M., 83

Kong, Z.-P., 85

Kratochví, B., 113

Królikowska, M., 103

Kuz'ma, Yu., 1

Laus, G., 77

Lefevre, C., 3

Li, X., 33, 36

Lin, J.-M., 93

Linker, T., 17, 19, 21

Linker, U., 19

Liu, G., 81

Liu, M.-C., 23

Liu, Q.-W., 25

Liu, Z., 101

Liu, Z.-J., 119

Mahjoub, A. R., 45, 47

Manríquez, V., 79, 87

Miyamoto, K., 31

Morsali, A., 45, 47

Murata, F., 31

Nakahodo, T., 27

Nöth, H., 107, 110

Nouryan Aval, S., 45

Nuss, H., 95

Nuss, J., 95

Ohno, M., 29

Oueslati, A., 105

Palvadeau, P., 71

Pang, Z., 85

Perrin, C., 116

Peters, E.-M., 17, 19, 21

Peters, K., 17, 19, 21

Piironen, A. J., 41

Pirelahi, H., 15

Polamo, M., 39, 41, 43

Polykova, L., 12

Popowski, E., 83

Pourayoubi, M., 65

Prieto, M., 5

Pu, S.-Z., 81

Rayes, A., 105

Rebien, F., 17

Reinke, H., 83

Rietz, I., 83
Rivera, P., 87

Rodríguez, M., 87

Roeper, S., 89

Roisnel, T., 116

Ruiz, D., 87

Rzaigui, M., 105

Salvadó, M. A., 5

Satke, J., 113

Schmidhammer, H., 77

Schöllhorn, B., 71

Schüllner, F., 77

Shariatinia, Z., 65

Shen, L., 81

Shi, L.-X., 93

Simon, A., 3

Syssa-Magalé, J.-L., 71

Takahashi, T. T., 27

Talaei, Z., 47

Talja, M., 39, 41, 43

Tan, C.-H., 25

Tao, X.-T., 101

Veremchuk, I., 1

Wang, D.-Y., 36

Wang, L., 101

Wang, R.-F., 58, 61

Wang, R.-J., 81

Wang, S.-L., 58, 61

Wartchow, R., 89

Watabe, M., 27

Wu, H.-Y., 23

Wu, W.-S., 93

Wurst, K., 77

Xiao, H.-P., 69

Xiao, Q., 81

Xu, J.-K, 81

Yang, F.-L., 119

Yang, J.-X., 101

Yong, $L, 49,53$

Yu, W.-T., 101

Yu, Y.-Y., 75

Yue, B., 85

Žabka, M., 113

Zeng, X.-R., 75

Zhang, B.-S., 73, 75

Zhang, G.-J., 97

Zhang, H.-G., 101

Zhang, L.-P., 69

Zhang, T., 25

Zhang, W.-G., 58, 61

Zhang, Z.-Y., 36

Zhao, G.-L., 99

Zhou, X.-G., 85

Zhu, D.-Y., 25

Zhu, L.-G., 69

Zou, Y.-Q., 33, 36 


\author{
$\mathrm{B}_{9} \mathrm{Ni}_{15} \mathrm{Yb}_{2}, 1$ \\ $\mathrm{BaO}_{4} \mathrm{Se}, 5$ \\ $\mathrm{Br}_{10} \mathrm{CoGd}_{6}, 3$ \\ $\mathrm{C}_{6} \mathrm{H}_{9} \mathrm{~N}_{3} \mathrm{O}_{3}, 45$ \\ $\mathrm{C}_{7} \mathrm{H}_{10} \mathrm{ClN}, 95$ \\ $\mathrm{C}_{7} \mathrm{H}_{11} \mathrm{Cl}_{2} \mathrm{NO}, 105$ \\ $\mathrm{C}_{8} \mathrm{H}_{11} \mathrm{~N}_{2} \mathrm{O}_{5} \mathrm{P}, 65$ \\ $\mathrm{C}_{10} \mathrm{H}_{14} \mathrm{Cl}_{2} \mathrm{~N}_{2} \mathrm{O}_{2} \mathrm{Pd}, 27$ \\ $\mathrm{C}_{10} \mathrm{H}_{14} \mathrm{O}_{9}, 19$ \\ $\mathrm{C}_{10} \mathrm{H}_{16} \mathrm{Cl}_{2} \mathrm{~N}_{4}, 103$ \\ $\mathrm{C}_{10} \mathrm{H}_{18} \mathrm{~N}_{4} \mathrm{O}_{2}, 103$ \\ $\mathrm{C}_{11} \mathrm{H}_{9} \mathrm{ClN}_{2}, 39$ \\ $\mathrm{C}_{13} \mathrm{H}_{12} \mathrm{O}_{2} \mathrm{~S}, 23$ \\ $\mathrm{C}_{14} \mathrm{H}_{9} \mathrm{~N}_{3} \mathrm{O}_{3}, 93$ \\ $\mathrm{C}_{14} \mathrm{H}_{27} \mathrm{KNO}_{6} \mathrm{PS}_{3}, 67$ \\ $\mathrm{C}_{15} \mathrm{H}_{22} \mathrm{O}_{2}, 87$ \\ $\mathrm{C}_{16} \mathrm{H}_{9} \mathrm{~F} 5 \mathrm{Fe}, 71$ \\ $\mathrm{C}_{16} \mathrm{H}_{15 \mathrm{Br}_{2} \mathrm{O}_{75}, 25}$ \\ $\mathrm{C}_{16} \mathrm{H}_{20} \mathrm{GdNO}_{11}, 99$
}

\author{
$\mathrm{C}_{16} \mathrm{H}_{21} \mathrm{NO}_{11}, 21$ \\ $\mathrm{C}_{17} \mathrm{H}_{12} \mathrm{Cl}_{2} \mathrm{~N}_{2} \mathrm{O}_{2} \mathrm{~Pb}, 73$ \\ $\mathrm{C}_{17} \mathrm{H}_{13} \mathrm{FN}_{2} \mathrm{OS}, 119$ \\ $\mathrm{C}_{17} \mathrm{H}_{14} \mathrm{Cl}_{3} \mathrm{~N}_{3} \mathrm{~W}, 43$ \\ $\mathrm{C}_{17} \mathrm{H}_{19} \mathrm{~N}_{3} \mathrm{NiO}_{3}, 97$ \\ $\mathrm{C}_{17} \mathrm{H}_{22} \mathrm{~N}_{2}, 41$ \\ $\mathrm{C}_{18} \mathrm{H}_{16} \mathrm{O}_{3}, 17$ \\ $\mathrm{C}_{18} \mathrm{H}_{34} \mathrm{Mn}_{2} \mathrm{~N}_{6} \mathrm{O}_{16}, 85$ \\ $\mathrm{C}_{21} \mathrm{H}_{17} \mathrm{~F}_{3} \mathrm{~N}_{3} \mathrm{O}_{6} \mathrm{ReS}, 107$ \\ $\mathrm{C}_{21} \mathrm{H}_{28} \mathrm{BN}_{2} \mathrm{O}_{2.50}, 89$ \\ $\mathrm{C}_{21} \mathrm{H}_{30} \mathrm{BN}_{2} \mathrm{O}_{2.50}, 89$ \\ $\mathrm{C}_{23} \mathrm{H}_{43} \mathrm{~N}_{3} \mathrm{O}_{10}, 113$ \\ $\mathrm{C}_{24} \mathrm{H}_{18} \mathrm{Cl}_{2} \mathrm{~N}_{4} \mathrm{O}_{5} \mathrm{Zn}, 47$ \\ $\mathrm{C}_{26} \mathrm{H}_{16} \mathrm{~F}_{2} \mathrm{~N}_{2} \mathrm{O}_{4} \mathrm{~Pb}, 75$ \\ $\mathrm{C}_{26} \mathrm{H}_{34} \mathrm{~N}_{2} \mathrm{NiO}_{4}, 31$ \\ $\mathrm{C}_{26} \mathrm{H}_{52} \mathrm{~N}_{2} \mathrm{Si}_{6}, 83$ \\ $\mathrm{C}_{29} \mathrm{H}_{16} \mathrm{~F}_{12} \mathrm{~S}_{2}, 81$ \\ $\mathrm{C}_{30} \mathrm{H}_{21} \mathrm{~F}_{3} \mathrm{OS}$, 15 \\ $\mathrm{C}_{30} \mathrm{H}_{24} \mathrm{~N}_{2}, 101$
}

$\mathrm{C}_{32} \mathrm{H}_{72} \mathrm{Br}_{14} \mathrm{Mo}_{6} \mathrm{~N}_{2}, 116$

$\mathrm{C}_{34} \mathrm{H}_{28} \mathrm{BrMnO}_{3} \mathrm{P}_{2}, 29$

$\mathrm{C}_{35} \mathrm{H}_{37} \mathrm{IN}_{2} \mathrm{O}_{3}, 77$

$\mathrm{C}_{38} \mathrm{H}_{32} \mathrm{Cd}_{2} \mathrm{~N}_{4} \mathrm{O}_{14} \mathrm{~S}_{2}, 69$

$\mathrm{C}_{38} \mathrm{H}_{42} \mathrm{~N}_{2} \mathrm{O}_{6}, 79$

$\mathrm{C}_{48} \mathrm{H}_{42} \mathrm{Dy}_{2} \mathrm{O}_{12}, 36$

$\mathrm{C}_{55} \mathrm{H}_{112} \mathrm{~K}_{3} \mathrm{~N}_{7} \mathrm{O}_{18} \mathrm{Sn}_{9}, 49$

$\mathrm{C}_{60} \mathrm{H}_{48} \mathrm{~F}_{6} \mathrm{~N}_{8} \mathrm{O}_{12} \mathrm{P}_{2} \mathrm{Re}_{2} \mathrm{~S}_{2}, 110$

$\mathrm{C}_{66} \mathrm{H}_{55} \mathrm{Ag}_{2} \mathrm{P}_{3} \mathrm{Se}_{2}, 58$

$\mathrm{C}_{72} \mathrm{H}_{58} \mathrm{Ho}_{2} \mathrm{~N}_{4} \mathrm{O}_{12}, 33$

$\mathrm{C}_{79} \mathrm{H}_{160 \mathrm{~K}} \mathrm{MoN}_{12} \mathrm{O}_{27} \mathrm{Sn} 9,53$

$\mathrm{C}_{84} \mathrm{H}_{70} \mathrm{Hg}_{4} \mathrm{P}_{2} \mathrm{Se}_{8}, 61$

$\mathrm{Cs}_{4} \mathrm{Se}_{8} \mathrm{Si}_{2}, 12$

$\mathrm{Cs}_{4} \mathrm{Se}_{10} \mathrm{Si}_{4}, 13$

GeKPrSe 4,7

GeKS Th, 7

$\mathrm{K}_{3} \mathrm{PSe}_{4}, 11$

$\mathrm{K}_{4} \mathrm{P}_{2} \mathrm{Se}_{6}, 9$ 